\title{
(C) Staffing and resource adequacy OPEN ACCESS strongly related to RNs' assessment of patient safety: a national study of RNs working in acute-care hospitals in Sweden
}

\author{
Lisa Smeds Alenius, Carol Tishelman, Sara Runesdotter, Rikard Lindqvist
}

- Additional material is published online only. To view please visit the journal online (http://dx.doi.org/10.1136/bmjqs2012-001734)

Department of Learning, Informatics, Management and Ethics, Medical Management Centre, Karolinska Institutet, Stockholm, Sweden

\section{Correspondence to}

Lisa Smeds Alenius, Department of Learning, Informatics, Management and Ethics, Medical Management Centre, Karolinska Institutet, Tomtebodavägen 18A, LIME/ MMC, floor 5, Stockholm S-171 77, Sweden; lisa.smeds@ki.se

Received 3 December 2012 Revised 12 September 2013 Accepted 14 September 2013 Published Online First 14 October 2013

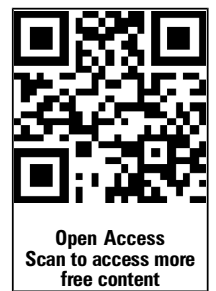

To cite: Smeds Alenius $L$, Tishelman C, Runesdotter S, et al. BMJ Qual Saf 2014;23:242-249.

\begin{abstract}
Introduction Although registered nurses (RNs)

are central in patient care, we have not found

prior research that specifically addresses how RNs

assess the safety of patient care at their

workplace and how factors in RNs' work environment are related to their assessments.

This study aims to address these issues.
\end{abstract}

Methods 9236 RNs working with inpatient care in 79 acute-care hospitals in Sweden completed a national population-based survey, including Practice Environment Scale of the Nursing Work Index-Revised and items from Agency for Healthcare Research and Quality's Hospital Survey on Patient Safety Culture. Correlation coefficients (Pearson and Spearman) and proportional odds regression were used for analysis.

Results Nursing work environment factors were strongly related to RNs' assessments of patient safety. RNs' perception of having adequate staffing and resources improved their assessment of patient safety by at least two and a half times (OR $2.74 \mathrm{Cl} 2.52$ to 2.97). RNs with a higher level of involvement in direct patient care gave a better patient safety grade than RNs with a more supervisory role. Most, but not all, patient safety culture items were related to RNs' assessed patient safety grade. We found that work experience seemed to have no influence on RNs patient safety assessment.

Conclusions While previous research emphasises patient-to-nurse ratios in strengthening patient safety practices, this study complements this by emphasising RNs' own perception of having enough staff and resources to provide quality nursing care, as well as having good collegial nurse-physician relations and the presence of visible and competent nursing leadership—all factors highly related to RNs' assessment of the safety of patient care at their workplace.

\section{INTRODUCTION}

Nurse-related factors such as staffing, education, collegial relations with physicians and leadership have all been shown to affect patient safety outcomes, for example, 30-day mortality, in-hospital falls, medication errors, pressure ulcers and hospital-acquired pneumonia. ${ }^{1-9}$ Nurses are the largest group of healthcare providers. ${ }^{10}{ }^{11}$ Their central role in patient care makes their perspective unique in assessing patient safety and care quality at their workplace. ${ }^{10}$ Reports from the US-based Institute of Medicine (IOM) emphasise a focus on the nursing work environment as a means to improve patient safety and quality care outcomes. ${ }^{10} 12$ While patient safety research often focuses on defining patient care errors and the patient safety concept as a whole, the perspective of the bedside nurse working at the frontline or 'sharp end' of patient safety practices also merits investigation. ${ }^{10}{ }^{13}$ Spears $^{14}$ argues the importance of obtaining a better understanding of the perspectives of those providing care to generate knowledge of the care environment where errors occur. However, we have not found prior research that specifically addresses how registered nurses (RNs) assess the safety of patient care at their workplace and how different nurse-related factors apply to their assessments.

In this study, we therefore aim to investigate how RNs' assessments of (a) the safety of patient care at their workplace, 
(b) the nursing work environment, (c) the patient safety culture, as well as (d) their level of involvement in direct patient care, and (e) length of work experience as an RN relate to, and interact with, RNs' global assessment of patient safety in acute-care hospitals in Sweden.

\section{METHOD}

The data analysed here derive from the Swedish subset of the EU 7th Framework Registered Nurse Forecasting (RN4CAST) database. RN4CAST included a multinational survey of RNs (described in depth in ref. ${ }^{15}$ ) based on 118 items derived from well-known and extensively validated instruments, ${ }^{16-20}$ as well as questions developed and tested in prior research that were translated for the study. ${ }^{3} 21$ Translations were validated using a content validity index $(\mathrm{CVI})^{22}$ giving a Scale-CVI score of 0.91 for the Swedish version of the survey. Ethical approval was received for the RN4CAST project by the regional Ethical Review Board in Stockholm (Dnr 2009/1587-31/5) prior to its initiation.

\section{Data collection}

In the RN4CAST survey, the included RNs were clinically active, working directly with inpatient medical or surgical care in acute-care hospitals. In Sweden, survey participants were recruited through the member register of the Swedish Association of Health Care Professionals, with $>80 \%$ of clinically active RNs in the country as members (Vårdförbundet [Swedish Association of Health Professionals] Per Malmquist, personal correspondence, 1 February 2011). The member register contains information on workplace, both hospital and department, but not on the RN's specific function or involvement in inpatient versus outpatient care. All RNs registered as working in medical or surgical departments were therefore recruited to receive the survey $(\mathrm{N}=33083)$. The survey was distributed between January and March 2010 by Statistics Sweden with the option of answering by web or paper. After three reminders, the return rate was $69.8 \%(\mathrm{n}=23087)$, with $12 \%$ of responses received via the web version of the survey.

The first survey questions were formulated to control inclusion criteria, that is, if the respondent was clinically active in direct inpatient adult medical or surgical care. At this stage, 12913 RNs were excluded since they did not meet inclusion criteria, for example, workplaces or functions beyond the scope of inclusion criteria for the study, giving a total study database of 10174 respondents. Due to internal attrition or invalid responses on the survey items that are analysed here, an additional $938 \mathrm{RN}$ responses were excluded. The responses of all the remaining 9236 RNs constitute the database for this article.

\section{Variables}

Explanatory variables

The three subscales-(1) staffing and resource adequacy, (2) collegial nurse-physician relations and (3) nurse manager ability, leadership and support of nurses-from the Practice Environment Scale of the Nursing Work Index-Revised (PES-NWI) ${ }^{16-18}$ have been used to investigate different dimensions of the nursing work environment on a four-point scale ranging from $1=$ strongly disagree to $4=$ strongly agree. The items included in each subscale are shown in table 1 . The PES-NWI subscales were analysed by calculating means for the individual $\mathrm{RN}$, ranging from 1 to 4 in each subscale. As noted above, observations with missing values in $>50 \%$ of the items in each subscale were omitted in the analysis $(n=148)$ to avoid the mean of the subscale for an individual nurse being determined by a single item. The reliability of the three subscales was tested using an internal consistency test, Cronbach's $\alpha$. All subscales had a Cronbach's $\alpha$ between 0.76 and 0.89 , which is consistent with prior research. ${ }^{17} 1823$

$R N$ work experience was investigated by the single question: "How many years have you worked as a registered nurse in your career?" $R N s$ ' level of involvement in direct patient care was assessed with the question: "How would you describe your role in caring for most of the patients on your most recent shift?" The three response alternatives were (a) "I provided most care myself", (b) "I supervised the care by others and provided some myself" and (c) "I provided only limited care such as dressing changes or drug administration and most of the direct care was done by others".

Seven items derived from the Agency for Healthcare Research and Quality's (AHRQ) Hospital Survey on Patient Safety Culture (HSOPSC) ${ }^{24} 25$ were used to investigate patient safety culture. Responses were given on a five-point Likert scale ranging from $1=$ strongly disagree to $5=$ strongly agree (items shown in table 1).

\section{Outcome variable}

Global patient safety grade is based on the responses to a single-item assessment, "Please give your unit/ ward an overall grade on patient safety", on a fivepoint scale ranging from $1=$ failing to $5=$ excellent. The global patient safety grade item comes from the HSOPSC instrument, which has been used and validated in several studies. ${ }^{24-27}$

Table 1 presents correlations between the individual explanatory variables and the global patient safety grade. The highest correlation was found for the item "The actions of hospital management show that patient safety is a top priority" (Spearman 0.44), with only two other items over 0.40 . All correlations were significant. 


\begin{tabular}{|c|c|c|}
\hline & $\begin{array}{l}\text { Patient safety grade } \\
\text { (Spearman correlation } \\
\text { coefficient) }\end{array}$ & $\begin{array}{l}\text { Reliability } \\
\text { (Cronbach's } \alpha \text { ) }\end{array}$ \\
\hline Staffing and resource adequacy (PES-NWI) & & 0.78 \\
\hline Adequate support services allow me to spend time with my patients & $0.27^{*}$ & \\
\hline Enough time and opportunity to discuss patient care problems with other nurses & $0.34^{*}$ & \\
\hline Enough registered nurses on staff to provide quality patient care & $0.43^{*}$ & \\
\hline Enough staff to get the work done & $0.41 *$ & \\
\hline Collegial nurse-physician relations (PES-NWI) & & 0.89 \\
\hline Physicians and nurses have good working relationships & $0.27^{*}$ & \\
\hline Physicians value nurses' observations and judgements & $0.20^{*}$ & \\
\hline Physicians recognise nurses' contributions to patient care & $0.20^{*}$ & \\
\hline A lot of team work between nurses and physicians & $0.22^{*}$ & \\
\hline Physicians respect nurses as professionals & $0.24^{*}$ & \\
\hline Collaboration between nurses and physicians & $0.26^{*}$ & \\
\hline Physicians hold nurses in high esteem & $0.23^{*}$ & \\
\hline Nurse manager ability, leadership and support of nurses (PES-NWI) & & 0.76 \\
\hline A supervisory staff that is supportive of nurses & $0.39^{*}$ & \\
\hline A nurse manager who is a good manager and leader & $0.30^{*}$ & \\
\hline Praise and recognition for a job well done & $0.29^{*}$ & \\
\hline $\begin{array}{l}\text { A nurse manager who backs up the nursing staff in decision making, even if the conflict is } \\
\text { with a physician }\end{array}$ & $0.27^{*}$ & \\
\hline \multicolumn{3}{|l|}{ RNs' level of involvement in direct patient care } \\
\hline How would you describe your role in caring for most of the patients on your most recent shift? & $-0.04^{*}$ & \\
\hline \multicolumn{3}{|l|}{ Patient safety culture (HSOPSC) } \\
\hline Staff feel like their mistakes are held against them & $-0.32^{*}$ & \\
\hline Important patient care information is often lost during shift changes & $-0.39^{*}$ & \\
\hline Things 'fall between the cracks' when transferring patients from one unit to another & $-0.35^{*}$ & \\
\hline Staff feel free to question the decisions or actions of those in authority & $0.27^{*}$ & \\
\hline In this unit, we discuss ways to prevent errors from happening again & $0.36^{*}$ & \\
\hline We are given feedback about changes put into place based on event reports & $0.32^{*}$ & \\
\hline The actions of hospital management show that patient safety is a top priority & $0.44^{*}$ & \\
\hline
\end{tabular}

Reliability of PES-NWI subscales is included.

${ }^{*} \mathrm{p}<0.0001$.

HSOPSC, Hospital Survey on Patient Safety Culture; PES-NWI, Practice Environment Scale of the Nursing Work Index; RNs, registered nurses.

Control variables

Individual nurse characteristics, education and sex were controlled for in the multivariate analysis. Since age was highly correlated with work experience (Pearson coefficient $0.8, \mathrm{p}<0.0001$ ), it was not controlled for in the multivariate model.

\section{Statistical analysis}

Descriptive statistics were used, including crosstabulations, frequencies and graphs, to discern any data anomalies, such as outliers or extreme values, and to describe the study participants. Correlation coefficient tests (Pearson and Spearman) were used to investigate correlations between items.

For multivariate analysis, a proportional odds model ${ }^{28}$ was used to estimate the effects of work environment, work experience as RN, RNs' level of involvement in direct patient care and patient safety culture on the global patient safety grade as the outcome. The proportional odds model has the advantage of treating the outcome of patient safety as an ordinal variable, not assuming equal distance between response points. ${ }^{28}$

First, bivariate regression models were computed for each of the explanatory variables and the global patient safety grade. Thereafter, a multivariate proportional odds model for the global patient safety grade was performed by including all explanatory variables and controlling for education and sex. Empirical logits, predictor plots and a score test were used to check the proportional odds assumptions of the models. CIs were set at 95\%. Data were analysed using SAS V.9.3.

\section{RESULTS}

\section{Descriptive statistics}

The 9236 RNs included in these analyses had a mean age of 39 years (median 38, SD 11), an average of 11 years' (median 8, SD 10) work experience as an RN 
Table 2 Descriptive statistics of the outcome measure and independent variables

\begin{tabular}{|c|c|c|c|c|c|c|c|c|c|c|}
\hline & & & & & & & & $\mathrm{n}$ & & $\%$ \\
\hline Outcome measure & & & & & & & & & & \\
\hline Please give your unit or ward an overall grade on patient safety & & & & & & & & & & \\
\hline Failing & & & & & & & & 191 & & \\
\hline Poor & & & & & & & & 865 & & 9 \\
\hline Acceptable & & & & & & & & 4545 & & 49 \\
\hline Very good & & & & & & & & 3356 & & 36 \\
\hline Excellent & & & & & & & & 279 & & 3 \\
\hline Explanatory variables & & & & & & & & & & \\
\hline How would you describe your role in caring for most of the patients on your mos & cent shif & & & & & & & & & \\
\hline I provided most care myself & & & & & & & & 2490 & & 27 \\
\hline I supervised the care by others and provided some myself & & & & & & & & 5098 & & 55 \\
\hline I provided only limited care such as dressing changes or drug administration ar & most of $t$ & e dir & ect care & vas $d$ & one by 0 & hers & & 1648 & & 18 \\
\hline & $\begin{array}{l}\text { Strong } \\
\text { disagr }\end{array}$ & & Disagr & & Neithe & & Agree & & $\begin{array}{l}\text { Stron } \\
\text { agree }\end{array}$ & \\
\hline & $\mathbf{n}$ & $\%$ & $\mathrm{~N}$ & $\%$ & $\mathbf{n}$ & $\%$ & $\mathrm{n}$ & $\%$ & $\mathbf{n}$ & $\%$ \\
\hline The following questions ask for your opinion about patient safety issues in your e & loyment & ettin & & & & & & & & \\
\hline Staff feel like their mistakes are held against them & 1682 & 18 & 2514 & 27 & 3317 & 36 & 1471 & 16 & 252 & 3 \\
\hline Important patient care information is often lost during shift changes & 1379 & 15 & 4196 & 45 & 2171 & 24 & 1353 & 15 & 137 & 1 \\
\hline $\begin{array}{l}\text { Things 'fall between the cracks' when transferring patients from one unit to } \\
\text { another }\end{array}$ & 473 & 5 & 2602 & 28 & 2919 & 32 & 2829 & 31 & 413 & 4 \\
\hline Staff feel free to question the decisions or actions of those in authority & 1111 & 12 & 2004 & 22 & 2724 & 29 & 3019 & 33 & 378 & 4 \\
\hline In this unit, we discuss ways to prevent errors from happening again & 241 & 3 & 913 & 10 & 1238 & 13 & 5268 & 57 & 1576 & 17 \\
\hline We are given feedback about changes put into place based on event reports & 751 & 8 & 1318 & 14 & 1737 & 19 & 3669 & 40 & 1761 & 19 \\
\hline The actions of hospital management show that patient safety is a top priority & 1413 & 15 & 1563 & 17 & 3028 & 33 & 2638 & 29 & 594 & 6 \\
\hline
\end{tabular}

and worked at 79 acute-care hospitals in Sweden. The majority of these primarily female RNs (93\%) had a bachelor's degree in nursing (59\%) (data not shown).

The majority of respondents reported their level of involvement in direct patient care as "supervising the care performed by others and providing some care themselves". Approximately one-quarter reported "providing most care themselves", while 18\% reported "providing limited care themselves, with most care provided by others" (see table 2).

Low-to-moderate correlations (Spearman -0.04 to 0.44 ) were found between the explanatory variables and the patient safety grade outcome (see table 1).

Almost half the responding RNs reported the global patient safety grade of their unit as acceptable. Over one-third gave a 'very good' or 'excellent' patient safety grade, while $11 \%$ graded patient safety at their unit as 'poor' or 'failing' (see table 2).

In four of the seven items (items a, c, d, g) assessing patient safety culture (see table 2), approximately one-third or more of the respondents were neutral (neither agreeing nor disagreeing with the statement). The proportion of neutral responses to the remaining three questions (items $\mathrm{b}, \mathrm{e}, \mathrm{f}$ ) varied from $13 \%$ to 24\%. Almost half the responding RNs disagreed (disagree or strongly disagree), while 19\% agreed (agree or strongly agree) with the statement "that their mistakes are held against them". Most RNs disagreed that "important patient care information often is lost during shift changes". There is an almost equal balance between RNs who disagreed and those who agreed with the item "things 'fall between the cracks' when transferring patients from one unit to another". While there is relative balance between RNs who disagreed and those who agreed with the statement "staff feel free to question the decisions or actions of those in authority", $12 \%$ of the responding RNs strongly disagreed with this statement, that is, reported not feeling free to question those in authority. The majority of RNs agreed with the statement that in their unit or workplace, they are "discussing ways to prevent errors from happening again". Most RNs also agreed they were "given feedback about changes put into place based on event reports". In the item regarding hospital management, there is relative balance between RNs who disagreed and agreed, but $15 \%$ strongly disagreed with the statement that "the actions of hospital management show patient safety is a top priority" (see table 2).

\section{Multivariate analysis}

The results of the multivariate analysis for nursing work environment, level of involvement in direct patient care, $\mathrm{RN}$ work experience and patient safety culture are shown in table 3. Three of the five explanatory variables with the highest odds of giving 
Table 3 Relationship between work environment, RN involvement in patient care, patient safety culture, work experience and the patient safety grade outcome

\begin{tabular}{|c|c|c|c|c|}
\hline & \multicolumn{2}{|c|}{$\begin{array}{l}\text { Unadjusted } \\
\text { (bivariate) } \\
\text { models }\end{array}$} & \multicolumn{2}{|c|}{$\begin{array}{l}\text { Adjusted } \\
\text { (multivariate) } \\
\text { models* }\end{array}$} \\
\hline & $\mathrm{OR}$ & $95 \% \mathrm{Cl}$ & OR & $95 \% \mathrm{Cl}$ \\
\hline \multicolumn{5}{|l|}{ Work environment (PES-NWI) } \\
\hline Staffing and resource adequacy (four items) & 5.44 & 5.06 to 5.85 & 2.74 & 2.52 to 2.97 \\
\hline Collegial nurse-physician relations (seven items) & 3.40 & 3.14 to 3.69 & 1.43 & 1.30 to 1.57 \\
\hline Nurse manager ability, leadership and support of nurses (four items) & 4.50 & 4.18 to 4.84 & 1.49 & 1.36 to 1.63 \\
\hline \multicolumn{5}{|l|}{ RNs' level of involvement in direct patient care } \\
\hline \multicolumn{5}{|l|}{ How would you describe your role in caring for most of the patients on your most recent shift? } \\
\hline I provided most care myself vs I supervised the care by others and provided some myself & 1.16 & 1.06 to 1.27 & 1.15 & 1.04 to 1.27 \\
\hline $\begin{array}{l}\text { I provided most care myself vs I provided only limited care such as dressing changes or drug } \\
\text { administration and most of the direct care was done by others }\end{array}$ & 1.28 & 1.13 to 1.44 & 1.19 & 1.04 to 1.35 \\
\hline $\begin{array}{l}\text { I supervised the care by others and provided some myself vs I provided only limited care such as dressing } \\
\text { changes or drug administration and most of the direct care was done by others }\end{array}$ & 1.10 & 0.99 to 1.22 & 1.03 & 0.92 to 1.16 \\
\hline \multicolumn{5}{|l|}{ Patient safety culture } \\
\hline Staff feel like their mistakes are held against them & 0.53 & 0.51 to 0.55 & 0.85 & 0.82 to 0.89 \\
\hline Important patient care information is often lost during shift changes. & 0.41 & 0.39 to 0.43 & 0.65 & 0.61 to 0.68 \\
\hline Things 'fall between the cracks' when transferring patients from one unit to another & 0.47 & 0.45 to 0.49 & 0.80 & 0.76 to 0.84 \\
\hline Staff feel free to question the decisions or actions of those in authority. & 1.67 & 1.61 to 1.73 & 1.01 & 0.97 to 1.06 \\
\hline In this unit, we discuss ways to prevent errors from happening again. & 2.24 & 2.14 to 2.35 & 1.27 & 1.20 to 1.35 \\
\hline We are given feedback about changes put into place based on event reports. & 1.75 & 1.69 to 1.82 & 1.04 & 0.99 to 1.09 \\
\hline The actions of hospital management show that patient safety is a top priority & 2.33 & 2.24 to 2.43 & 1.51 & 1.44 to 1.58 \\
\hline \multicolumn{5}{|l|}{ Work experience } \\
\hline Work experience as a nurse (by increment of 5) & 1.07 & 1.05 to 1.09 & 1.01 & 0.98 to 1.04 \\
\hline
\end{tabular}

${ }^{*}$ Adjustments were made for gender and education (not shown).

PES-NWI, Practice Environment Scale of the Nursing Work Index; RNs, registered nurse.

a better patient safety grade are related to the nursing work environment, that is, the better the RNs rated their work environment, the better patient safety grade they gave their unit or ward.

Nursing work environment

As noted, the three PES-NWI subscales were each averaged, with means ranging from 1 to 4 . This means that for a one-step increase in the subscale, the odds indicate the probability of scoring the patient safety grade one full scale step better using the mean.

The subscale staffing and resource adequacy was associated with an increase in RN-reported patient safety by a factor of 2.74 (OR). Nurse manager ability, leadership and support of nurses was related to a $51 \%$ increase (OR 1.49), while collegial nurse-physician relations was associated with a $43 \%$ increase (OR 1.43) in RN-reported patient safety.

Patient safety culture

In the patient safety culture items, two of the positively worded items were statistically significant. The item "The actions of hospital management show that patient safety is a top priority" showed the largest increase in RN-reported patient safety (OR 1.51), followed by the item "In this unit we discuss ways to prevent errors from happening again" (OR 1.27).
The statement that "important patient care information often is lost during shift changes" was associated with a decrease in RN-reported patient safety by a factor of 0.65 (OR). Statistically significant odds of giving a lower patient safety grade were also seen for the statements "things 'fall between the cracks' when transferring patients from one unit to another" (OR 0.80 ) and "Staff feel like their mistakes are held against them" (OR 0.85) (see table 3).

\section{RNs' involvement in direct patient care}

In the case of $\mathrm{RN}$ involvement in direct patient care, the comparison between the least active role in direct patient care ("I provided only limited care such as dressing changes or drug administration and most of the direct care was done by others") and the most active role in direct patient care ("I provided most care myself") was associated with a $19 \%$ increase in $\mathrm{RN}$-assessed patient safety if the $\mathrm{RN}$ reported providing most care herself (see table 3).

Length of RN work experience

Length of RN work experience shows no effect on RNs' assessment of patient safety in their workplace in the adjusted multivariate analysis. 


\section{DISCUSSION}

In this national population-based study of $9236 \mathrm{RNs}$ working in inpatient care in all 79 acute-care hospitals in Sweden, we found that nursing work environment factors are strongly related to RNs' own assessments of patient safety. In these data, the RNs' perception of having adequate staffing and resources increased their assessment of patient safety by at least two and a half times (per scale step). We found that RNs with more involvement in direct patient care gave a better patient safety grade than RNs with a more supervisory role. We also found that the majority, but not all, of the patient safety culture items were related to the $\mathrm{RN}$-assessed patient safety grade and that work experience seems to have no statistical influence on this assessment.

This study is part of a larger multinational study, RN4CAST, conducted within the EU 7th Framework Program. This involves both benefits and potential limitations. In some cases, survey items may lack specificity in the Swedish context, but on the other hand, the RN4CAST project design allows benchmarking with other settings and comparison with previous research. The items used in this analysis were all relevant in the Swedish setting. While another potential limitation of the study is that RNs included in the Swedish RN4CAST project were sampled through the Swedish Association of Health Care Professionals, it should be noted that $>80 \%$ of RNs are members of this organisation. The return rate of nearly $70 \%$ gives good coverage of clinically active RNs working with inpatient care in Sweden. Other sampling strategies, for example, through hospital management, as was the case in most other RN4CAST countries, involve other potential, although different, biases. The RN4CAST project focuses on inpatient care in medical or surgical departments in acute-care hospitals, although when considering the applicability of the results it should be remembered that other areas such as intensive care, labour and delivery, psychiatric wards and paediatric care are excluded. This Swedish study is unique not only because of its scope and in using a national sample of RNs representing all acutecare hospitals in the country but also because of the limited prior research in Sweden in this area.

Our study shows that certain aspects of the nursing work environment highly affect how RNs assess patient safety at their unit or workplace. We find it notable that over a third of the RNs chose a 'neutral' response in several of the individual patient safety culture items, although this survey data do not allow us to better probe the reasons for this. On the other hand, from an organisational perspective, one might consider positive responses as favourable and other responses-negative or neutral-as indicative of a need for improvement. We find that positive $\mathrm{RN}$ reports of having enough staffing and resources to discuss nursing issues with other RNs, to get the work done, and to deliver quality care, as well as having good collegial nurse-physician relations, and visible and competent nursing leadership are all highly related to RNs grading overall patient safety as better.

This last aspect is supported by the IOM report, ${ }^{10}$ which also emphasises the importance of strong, visible nursing leadership in creating a positive work environment and a 'culture of safety'. Several earlier studies have found adequate staffing to be linked to lower 30-day mortality. ${ }^{3} 629$ In a recent study, Aiken $e t a l^{1}$ link adequate nurse staffing and better-educated nurses in combination with a good work environment to decreased patient mortality, whereas hospitals with poor work environments were found to have the opposite outcome. In line with our study results, good nurse-physician relations and teamwork have been shown in a number of other studies to be positively related to patient safety outcomes. ${ }^{7} 30$

Our findings, along with the results of prior research in other settings, emphasise the importance of a work environment that enables RNs to feel safe and confident when providing care. The perception of having enough staff and resources may not be consistent with actual patient:staff ratio, but it appears to be an important factor related to how RNs view patient safety at their ward or unit.

Length of RN work experience is a more complex issue. Despite our preconceived assumption that experience might be an important factor in patient safety assessments, in this study we found no effect of the length of RN work experience on RNs' assessment of patient safety at their ward or unit. Other research on the relationship between work experience and safety outcomes shows mixed results. Some studies find a positive relationship between longer work experience and patient safety and safety practices, ${ }^{31-33}$ whereas other studies find these relationships are weak or non-existent. ${ }^{34} 35$ The studies differ in design, concept instrumentalisation, measurement tools and size; in addition, work experience is generally used as a control variable rather than as an explanatory variable in itself. In our study, work experience might be seen as a mediator since it shows a significant effect in the bivariate analysis but not in the multivariate analysis. It might be that the potential effect of work experience is covariant with some other variable that is absorbed by the 'true' explanatory variable in the multivariate analysis. It seems that work experience might be an important factor and may influence aspects of patient safety, but that this issue warrants further investigation.

Another intriguing finding in our study is that, in the multivariate analysis, three of the seven items assessing patient safety culture were not significantly related to RNs' assessment of global patient safety. This raises the question of whether the single-item global patient safety grade may say more about the assessments of nursing work environment than about patient safety per se. The use of this single patient safety item in RN4CAST is aimed to capture clinically active RNs' views of patient 
safety at their workplace. This item reflects not only the care they themselves provide but also allows an overview of their perspective of the safety of the care provided in general at their nursing ward. Single global items are often used in research settings, for example, in assessing quality of life, quality of care and other broad concepts. Single items have the benefit of being easy to administer and to answer, which potentially may give a higher response rate, and can be said to be all-encompassing, as they require respondents to consider their situation, choose what is relevant and not relevant, and then provide a single rating. ${ }^{36-38}$ One potential disadvantage of using a global item might be that it is difficult to know exactly what RNs include in their view of the patient safety concept. However, during the translation process of the full RN survey, seven think-aloud interviews were performed, in which RNs who had answered the survey were asked, among other things, what they considered when answering the patient safety item. All these RNs answered that they considered the care provided at their ward, not only the care they provided themselves. This points to the importance of further investigation into not only into the subjective measure of $\mathrm{RN}$-assessed overall patient safety but also into its relation to objective patient safety outcomes.

\section{CONCLUSION}

In this study, we investigated how individual RN factors and work environment factors relate to RNs' own assessment of patient safety at their workplace, finding that RNs' assessment of overall patient safety grades was related to their own perceptions of having enough staffing and resources to deliver quality nursing care, as well as having good collegial nursephysician relations, and the presence of visible and competent nursing leadership. While research emphasises patient-to-nurse ratios in strengthening patient safety practices in hospital care, ${ }^{1-5}$ it is important to investigate RNs' experiences of their work setting as they are at the 'sharp end' of patient care and are an instrumental part of patient safety work. RNs work at the patient's bedside, providing 'round-the-clock' care; it is therefore vital to know more about their practice environment to ensure good and safe care. Identifying work environment factors that are linked to patient safety adds to a systems approach to solving patient safety problems rather than using individual error events as the source for solutions.

Acknowledgements Special thanks to all participating RNs who generously shared their experiences. We also thank the Swedish Association of Health Care Professionals' support for data collection and their collaborative interest from project initiation and onwards, expressed especially through the efforts of Lisbeth Löpare Johansson, Ingrid Frisk and Per Malmquist.

Contributors All authors were involved in conception and design of this study, as well as analysing and interpreting the data. LSA, RL and CT drafted the manuscript. All authors have been involved in reviewing and editing the manuscript, and have approved the final version of the manuscript prior to publication.
Funding This research was funded by the EU 7th Framework Program (FP7/2007-2013) under grant agreement no. 223468, Karolinska Institutet's National Research School of Health Care Sciences, the Swedish Council for Working Life and Social Research, the Karolinska Institutet Strategic Research Programme in Care Sciences, the Swedish Association of Health Professionals, the regional agreement on medical training and research (ALF) between Stockholm County Council and Karolinska Institutet.

\section{Competing interests None.}

Ethics approval The regional Ethical Review Board in Stockholm.

Provenance and peer review Not commissioned; externally peer reviewed.

Data sharing statement For questions about additional material or data sharing, please contact the corresponding author.

Open Access This is an Open Access article distributed in accordance with the Creative Commons Attribution Non Commercial (CC BY-NC 3.0) license, which permits others to distribute, remix, adapt, build upon this work noncommercially, and license their derivative works on different terms, provided the original work is properly cited and the use is non-commercial. See: http://creativecommons.org/licenses/by$\mathrm{nc} / 3.0 /$

\section{REFERENCES}

1 Aiken LH, Cimiotti JP, Sloane DM, et al. Effects of nurse staffing and nurse education on patient deaths in hospitals with different nurse work environments. Med Care 2011;49:1047-53.

2 Kane RL, Shamliyan TA, Mueller C, et al. The association of registered nurse staffing levels and patient outcomes. Systematic review and meta-analysis. Med Care 2007;45:1195-204.

3 Aiken LH, Clarke SP, Sloane DM, et al. Hospital nurse staffing and patient mortality, nurse burnout, and job dissatisfaction. JAMA 2002;288:1987-93.

4 Needleman J, Buerhaus P, Mattke S, et al. Nurse-staffing levels and the quality of care in hosptials. N Engl J Med 2002;346:1715-22.

5 Duffield C, Diers D, O'Brien-Pallas L, et al. Nursing staffing, nursing workload, the work environment and patient outcomes. Appl Nurs Res 2011;24:244-55.

6 Tourangeau AE, Doran DM, Hall LM, et al. Impact of hospital nursing care on 30-day mortality for acute medical patients. J Adv Nur 2007;57:32-44.

7 Estabrooks C, Midodzi W, Cummings G, et al. The impact of hospital nursing characteristics on 30-day mortality. Nurs Res 2005;54:74-84.

8 Cummings GG, Midodzi WK, Wong CA, et al. The contribution of hospital nursing leadership styles to 30-day patient mortality. Nurs Res 2010;59:331-9.

9 Wong CA, Cummings GG. The relationship between nursing leadership and patient outcomes: a systematic review. J Nurs Manag 2007;15:508-21.

10 Page A, ed. Keeping patients safe: Transforming the work environment of nurses. Washington: National Academies Press, 2004.

11 Socialstyrelsen. Statistikdatabasen-Hälso- och sjukvårdspersonal http://www.socialstyrelsen.se/statistik/statistikdatabas (accessed 25 Oct 2012).

12 Institute of Medicine (IOM). The future of nursing-leading change, advancing health. Washington: National Academies Press, 2011.

13 Reason J. Understanding adverse events: human factors. Qual Health Care 1995;4:80-9. 
14 Spears P. Managing patient care error: nurse leaders perspectives. J Nurs Adm 2005;35:223-24.

15 Sermeus W, Aiken LH, Van den Heede K, et al. Nurse forecasting in Europe (RN4CAST): rationale, design and methodology. BMC Nurs 2011;10:6.

16 Aiken LH, Patrician PA. Measuring organizational traits of hospitals: the Revised Nursing Work Index. Nurs Res 2000;49:146-53.

17 Lake ET. Development of the practice environment scale of the Nursing Work Index. Res Nurs Health 2002;25:176-88.

18 Li Y-F, Lake ET, Sales AE, et al. Measuring nurses' practice environments with the revised nursing work index: evidence from registered nurses in the Veterans Health Administration. Res Nurs Health 2007;30:31-44.

19 Maslach C, Jackson SE. The measurement of experienced burnout. J Occup Behav 1981;2:99-113.

20 Lake ET. The nursing practice environment: measurement and evidence. Med Care Res Rev 2007;64(2 Suppl):104S-22S.

21 Aiken LH, Clarke SP, Sloane DM, et al. Effects of hospital care environment on patient mortality and nurse outcomes. J Nurs Adm 2008;38:223-9.

22 Polit DF, Beck CT. The content validity index: are you sure you know what's being reported? Critique and recommendations. Res Nur Health 2006;29:489-97.

23 Fuentelsaz-Gallego C, Moreno-Casbas MT, Gonzalez-Maria E. Validation of the Spanish version of the questionnaire Practice Environment Scale of the Nursing Work Index. Int J Nurs Stud 2013;50:274-80.

24 Sorra JS, Dyer N. Multilevel psychometric properties of the AHRQ hospital survey on patient safety culture. BMC Health Serv Res 2010;10:199.

25 Sorra J, Nieva V. Hospital survey on patient safety culture. (Prepared by Westat, under Contract No. 290-96-0004). AHRQ Publication No. 04-0041. Rockville, MD: Agency for Healthcare Research and Quality, 2004.

26 Aiken LH, Sermeus W, Van den Heede K, et al. Patient safety, satisfaction, and quality of hospital care: cross sectional surveys of nurses and patients in 12 countries in Europe and the United States. BMJ 2012;344(mar20 2):e1717-e17.

27 Mardon RE, Khanna K, Sorra J, et al. Exploring relationships between hospital patient safety culture and adverse events. J Patient Saf 2010;6:226-32.

28 Gameroff MJ. Using the Proportional Odds Model for Health-Related Outcomes: why, When, and How with Various SAS ${ }^{\circledR}$ Procedures. SUGI 30. Philadelphia, US, 2005.

29 Aiken LH, Smith HL, Lake ET. Lower medicare mortality among a set of hospitals known for good nursing care. Med Care 1994;32:771-87.

30 Gunnarsdóttir S, Clarke SP, Rafferty AM, et al. Front-line management, staffing and nurse-doctor relationships as predictors of nurse and patient outcomes. a survey of Icelandic hospital nurses. Int J Nur Stud 2009;46:920-7.

31 Raftopoulos V, Savva N, Papadopoulou M. Safety culture in the maternity units: a census survey using the Safety Attitudes Questionnaire. BMC Health Serv Res 2011;11:238.

32 Ramanujam R, Abrahamson K, Anderson J. Influences on nurse perceptions of hospital unit safety climate: an HLM approach. RCHE Publications, 2007. http://docs.lib.purdue.edu/rche_rp/34.

33 Elfering A, Semmer NK, Grebner S. Work stress and patient safety: observer-rated work stressors as predictors of characteristics of safety-related events reported by young nurses. Ergonomics 2006;49:457-69.

34 Reagan JT, Slechta AM. Factors related to radiation safety practices in California. Radiol Technol 2010;81:538-47.

35 Vecchio N, Scuffham PA, Hilton MF, et al. Work-related injury in the nursing profession: an investigation of modifiable factors. J Adv Nurs 2011;67:1067-78.

36 Sloan JA, Aaronson N, Cappelleri JC, et al. Assessing the clinical significance of single items relative to summated scores. Mayo Clin Proc 2002;77:479-87.

37 Youngblut JM, Casper GR. Single-item indicators in nursing research. Res Nurs Health 1993;16:459-65.

38 Nagy MS. Using a single-item approach to measure facet job satisfaction. J Occup Organ Psychol 2002;75:77-86. 